\title{
The Onset of Chromospheres in A-type Stars: The Altair Affair
}

\author{
S. Catalano ${ }^{1}$, P. Gouttebroze ${ }^{2}$, E. Marilli ${ }^{3}$, \\ R. Freire Ferrero ${ }^{2,4}$ \\ ${ }^{1}$ Istituto di Astronomia, Università di Catania, Viale A. Doria 6, \\ I-95125 Catania, Italy \\ ${ }^{2}$ Institut d'Astrophysique Spatiale, IAS/LPSP CNRS, BP Nº 10 , \\ 91371 Verrieres-le Buisson, France \\ ${ }^{3}$ Osservatorio Astrofisico di Catania, Viale A. Doria 6, I-95125 Catania, \\ Italy \\ ${ }^{4}$ Observatorie Astronomique, 11, Rue de l'Université, 67000 Strasbourg, \\ France
}

\begin{abstract}
Here we present preliminary results on the study of the chromosphere of Altair from $L \alpha$ high dispersion profiles. We report also the detection of $L \alpha$ chromospheric emission from $\alpha \operatorname{Cep}(\mathrm{A} 7 \mathrm{~V})$ and $\beta \operatorname{TrA}(\mathrm{F} 0 \mathrm{~V})$ with the IUE. We show that chromosphere may exist up to $B-V=0.22$. The possible heating mechanism, magnetic or nonmagnetic, for the chromosphere of these stars is also discussed.
\end{abstract}

\section{Introduction}

Several studies of activity in F stars, mainly based on the detection of the UV emission lines C II, C IV and $L \alpha$ and of the optical He I $5876 \AA$ absorption line (see Wolff et al., 1986; Simon and Landsman, 1987; Walter and Schrijver, 1987), place the onset of stellar chromospheric activity near $B-V=0.28$, which corresponds approximately to spectral type F0. However, X-ray observations of A and F stars (Schmitt et al., 1985) show that coronal activity may occur at a significantly earlier spectral type. With the exception of the $L \alpha$ in Altair (A7 V-IV, $B-V=0.22$ ) (Blanco et al., 1980), no significant chromospheric or transition region emission has been detected in these earlier type stars. In a study of $L \alpha$ emission, using low dispersion IUE spectra, Simon and Landsman (1987) did confirm the detection of emission from Altair but did not find any emission in the spectra of 10 other late A-type stars. 
Should one consider Altair a special case? Has Altair a solar type chromosphere? In an effort of answering these questions and defining more clearly the location of the high temperature boundary of chromospheric detection we have started an observing program of high dispersion $L \alpha$ spectra with IUE. Here we report some preliminary results on the chromospheric model of Altair and $L \alpha$ observations of Altair-like stars.

\section{Altair's chromosphere}

The absence of detectable transition region emission lines connected with the detection of X-ray and $L \alpha$ emission, poses the question of whether or not Altair has a solar type chromosphere. We have two basic tests to answer this question:

i) Analyse the $L \alpha$ profile: The $L \alpha$ profile has been obtained by different authors from high dispersion IUE spectra. However the main problem in defining the $L \alpha$ profile from that spectra, is the high geocoronal contamination. We have solved this problem making specific observations of the sky background, which allow us to make a correct cleaning of the stellar profile (Catalano et al., 1990). The emission profile ${ }^{1}$ we have obtained as average of four different spectra is quite similar to the solar $L \alpha$ profile suggesting a similar chromospheric structure.

ii) Calculate chromospheric models: We are computing $L \alpha$ profile from model atmospheres. The calculations are performed with the following assumptions: 1) NLTE; 2) partial redistribution; 3) hydrogen atom with five level plus continuum; 4) rotational broadening $v \sin i=220 \mathrm{~km} / \mathrm{sec}$; 5) $\mathrm{HI}$ interstellar absorption. The main characteristic of the models giving the best agreement with the observed profile is a temperature increase after a minimum value and a large plateau, i.e. a basically solar-type chromospheric structure.

\section{Are there other Altairs?}

We have already obtained high resolution, long exposure IUE spectra for two stars of spectral type very close to that of Altair: $\alpha$ Cep (A7 V) and $\beta \operatorname{TrA}(F 0-F 2 V)$. Even though the spectra are rather noisy, the $L \alpha$ emission component is clearly detected. These profiles including the averaged Altair profile are shown in Fig. 1. The over-imposed thin curve represents a best-fit gaussian profile, which has been used to make a preliminary rough estimate of the energy released in that line.

The integrated $L \alpha$ emission luminosities are given in Table 1 , together with the relevant parameters of the three stars. $\beta \operatorname{Tr} A$, which is of a little later spectral type, has a $L \alpha$ luminosity larger by a factor of about two than Altair, as it would be expected from the deeper convection zone. $\alpha$ Cep, with parameters of spectral type and rotation speed that make it just a "twin" of Altair, has a $L \alpha$ luminosity

${ }^{1}$ Based on observations by the IUE, collected at the Villafranca Satellite Tracking Station of the ESA. 

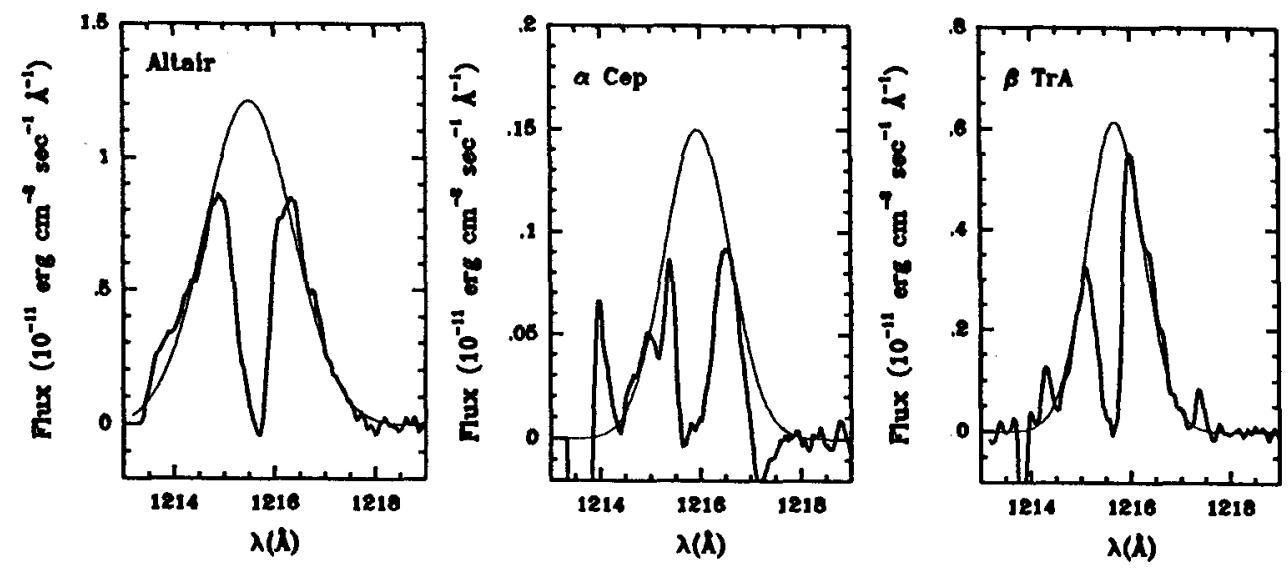

Fig. 1. Smoothed $L \alpha$ profiles of Altair, $\alpha$ Cep and $\beta$ TrA (solid lines) characterized by a deep interstellar HI absorption. The thin lines are gaussian curves fitted to the external profiles.

in very good agreement with Altair. The $L \alpha$ luminosities of Altair and $\alpha$ Cep, although are close to the lowest luminosity values of later spectral type stars, do not indicate a clear fading of chromospheric emission toward hot stars. From such few data we are not yet able to establish whether there is a sharp boundary or a smooth disappearance of chromospheres.

Table 1.

\begin{tabular}{ccccc}
\hline star & SP & $B-V$ & $v \sin i[\mathrm{~km} / \mathrm{sec}]$ & $\log L_{L \alpha}$ \\
\hline Altair & A7V-IV & 0.22 & 220 & 28.89 \\
$\alpha$ Cep & A7V & $0.22(0.24)$ & 245 & 28.77 \\
$\beta$ TrA & F0-F2V & 0.29 & 90 & 29.17 \\
\hline
\end{tabular}

\section{Nature of chromospheric heating}

Although it is generally agreed that chromospheric and coronal emission in late type stars is due to magnetic activity, the suggestion that magnetic dynamos exist in stars as early as A7 is still controversial. Theoretical models suggest that magnetic dynamos cannot be sustained in stars earlier than F6 (Durney and Latour, 1978). As a matter of fact simple evaluation shows that the Rossby number is about 20 for stars like Altair, while theory requires a value smaller than 1 to have an efficient dynamo. On the other hand Mangeney and Praderie (1984) have shown 
that magnetic dynamos can be efficient if a local Rossby number is defined. The break-down of the X-ray emission-rotation correlation and the absence of rotation braking for spectral types earlier than F5, have been invoked in support of a drop of magnetic dynamos in those stars (Walter, 1983). However Schmitt et al. (1985) from a larger sample of X-ray data have shown that a dependence of X-ray luminosity on the Rossby number may exist, and concluded that the magnetic activity is responsible for the heating of chromospheres and corona even in late A type stars.

Magnetic and acoustic heating may coexist in late type stars (Ulmschneider 1990). However it is very difficult or impossible to separate the two contributions in the observed emission fluxes. Schrijver (1987) suggests that there exists a "basal" flux in the chromospheric emission lines, of non-magnetic origin, which is negligible in the X-ray emission. The basal flux is also negligible in late type stars. He shows that the excess chromospheric flux, above the basal flux, is well correlated with the X-ray flux, while the total emission flux is not. Let us adopt this simple model to determine whether or not the chromospheres and coronae of our Altairlike stars are magnetically heated. In Fig. 2 we have plotted X-ray luminosity against $L \alpha$ luminosity for a sample of late type stars for which both quantities are available. From the figure we see that Altair and $\alpha \mathrm{Cep}$, for which only an upper-limit on the $\mathrm{X}$-ray flux is available, lie well below the regression line of late type stars. If we assume that the $\mathrm{X}$-ray emission is completely magnetic origin and that the chromospheric $L \alpha$ emission results from a combination of magnetic and non magnetic heating we can estimate the former as the value one would expect for a star situated on the regression line. It turns out that only about $10 \%$ of the $L \alpha$ emission in Altair, and perhaps also in $\alpha$ Cep, can be accounted for by magnetic heating. The largest amount should be of non magnetic (acoustic?) origin. Calculations of acoustic flux show that for these spectral type there is plenty of energy available to heat the chromosphere (Bohn, 1984). However detailed chromospheric models and energy balance calculations are needed to clarify this problem.

\section{Conclusions}

We have shown from the analysis of high dispersion $L \alpha$ profiles and preliminary atmospheric models that Altair has a solar type chromosphere. In addition we have found that Altair is not an unique case, because, at least its "twin" star $\alpha$ Cep possesses a chromosphere, as signalled by the detection of $L \alpha$ emission. These results definitely extend the presence of chromospheres up to $B-V=0.22$. Finally we have shown that the chromospheric $L \alpha$ emission can mostly be of non-magnetic origin.

Acknowledgements. This work has been possible thanks to the support of an international cooperation programme between the CNR (Italy) and DRCI-CNRS (France). The work was also supported by the MURST through the University of Catania, the Osservatorio 


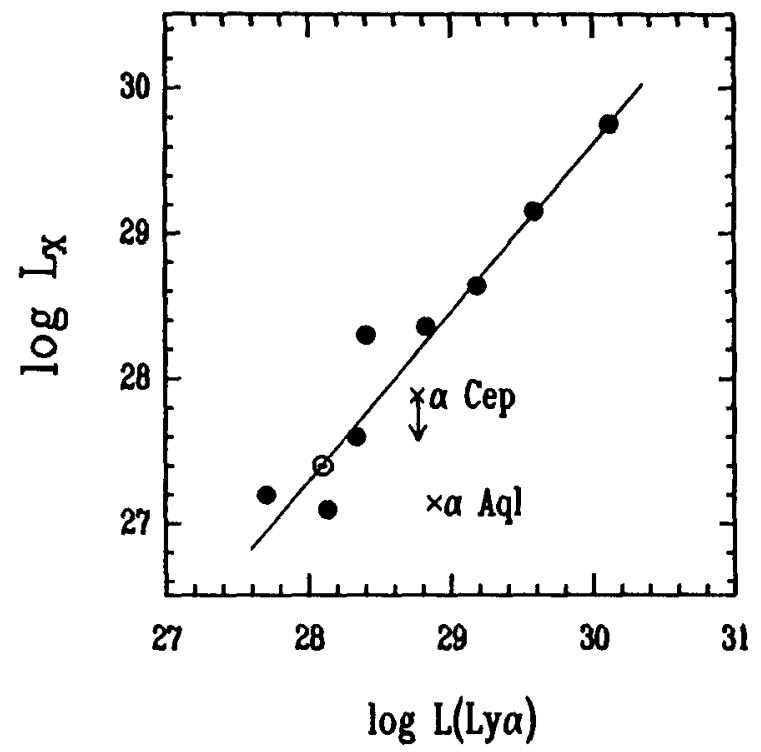

Fig. 2. Logarithms of X-ray luminosities vs. L $\alpha$ luminosities. Late-type stars (filled points) and the Sun define the best-fit line.

Astrofisico di Catania and the GNA (CNR). The extensive use of the computer facilities of the Catania ASTRONET site is also acknowledged.

\section{References}

Blanco, C., Catalano, S., Marilli E.: 1980, ESA SP-157, p. 67

Bohn, H.U.: 1984, Astron. Astrophys. 136, 338

Catalano, S., Marilli, E., Freire-Ferrero, R., Gouttebroze, P.: 1990, Astron. Astrophys., submitted

Durney, B.R., Latour, J.: 1978, Geophys. Astrophys. Fluid Dyn. 8, 241

Mangeney, A., Praderie, F.: 1984, Astron. Astrophys. 130, 143

Schmitt, J.H.M.M., Golub, L., Harnden, F.H.Jr., Maxon, C.W., Vaiana, S.G.: 1985, Astrophys. J. 290, 307

Schrijver, C.J.: 1987, in Cool Stars, Stellar Systems and the Sun, eds. J.L.Linsky, R.E., Stencel, Springer-Verlag, Berlin, p. 241

Simon, T., Landsman, W.: 1987, in Cool Stars, Stellar Systems and the Sun, eds. J.L. Linsky, R.E. Stencel, Springer-Verlag, Berlin, p. 265

Walter, F.M.: 1983, Astrophys. J. 274, 794

Walter, F.M., Schrijver, C.J.: 1987, in Cool Stars, Stellar Systems and the Sun, eds. J.L. Linsky, R.E. Stencel, Springer-Verlag, Berlin, p. 262

Ulmschneider, P.: 1990, in Mechanism of Chromospheric and Coronal Heating, eds. P. Ulmschneider et al., Springer, Berlin (in press)

Wolff, S.C., Boesgaard, A.M., Simon, T.: 1986, Astrophys. J. 310, 360 